Supporting Information

\title{
Antibacterial Adhesion of Poly(methyl methacrylate) Modified by Borneol Acrylate
}

Xueli Sun, ${ }^{\dagger}$ Zhiyong Qian, ${ }^{\ddagger}$, Lingqiong Luo, $^{\dagger}$ Qipeng Yuan, ${ }^{\dagger}$ Ximin Guo, ${ }^{\ddagger}$, Lei Tao, ${ }^{\|}$Yen Wei, and Xing Wang*,

${ }^{\dagger}$ College of Life Science and Technology, Beijing University of Chemical Technology, Beijing 100029, P. R. China

Key Laboratory for Biomechanics and Mechanobiology of Ministry of Education, School of Biological Science and Medical Engineering, Beihang University, Beijing, 100191, P. R. China

${ }^{\S}$ Department of Advanced Interdisciplinary Studies, Institute of Basic Medical Sciences, Beijing, 100850, P. R. China

" Department of Chemistry, Tsinghua University, Beijing 100084, P. R. China

X.S. and Z.Q. contributed equally to this work.

* E-mail: wangxing@mail.buct.edu.cn 


\section{Copolymer section}

\subsection{GPC characterization of copolymers}
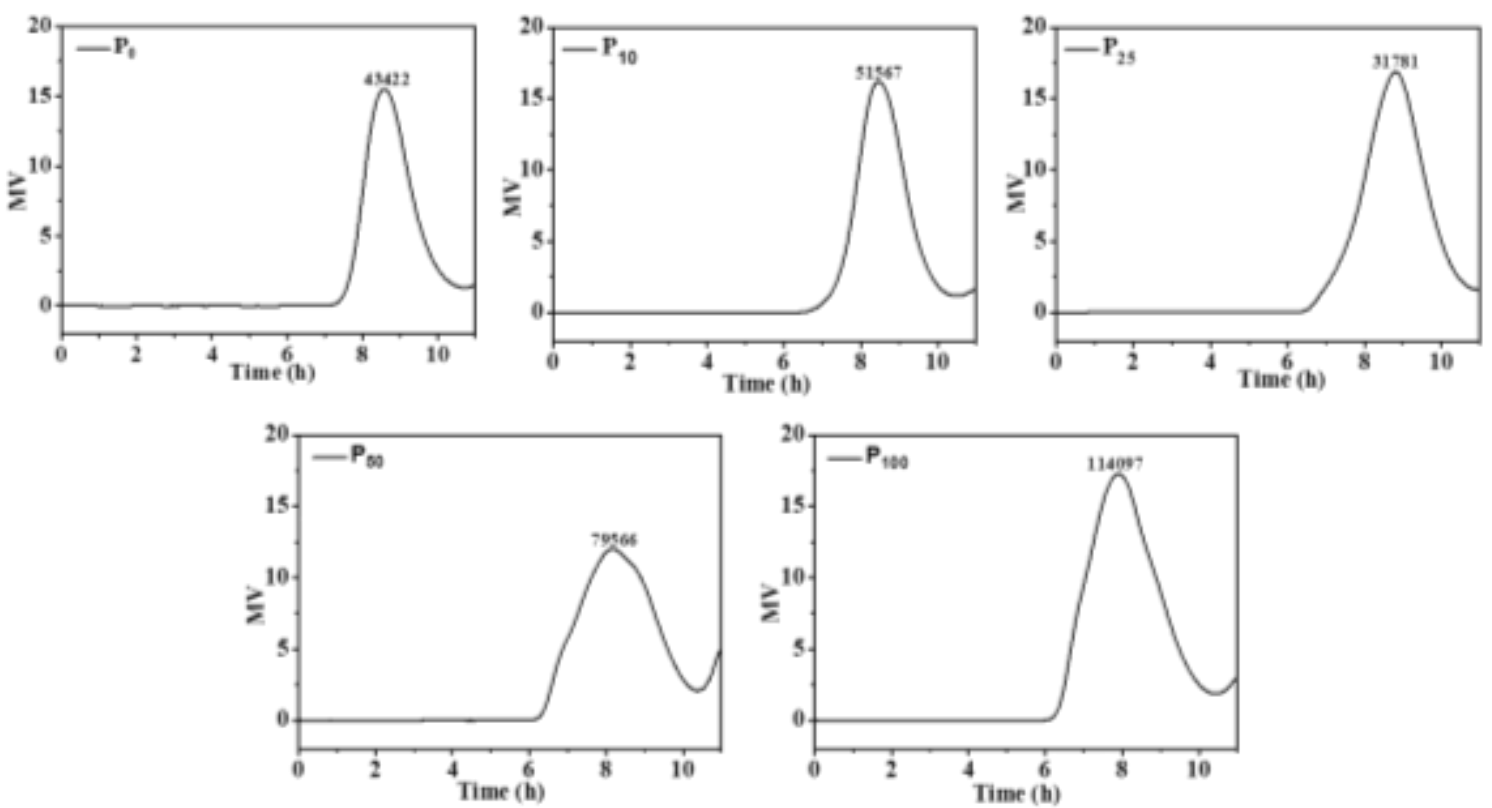

Figure S1. GPC spectra of $\mathrm{P}_{0}, \mathrm{P}_{10}, \mathrm{P}_{25}, \mathrm{P}_{50}$ and $\mathrm{P}_{100}$.

Table S1. GPC data of P(MMA-co-BA)s

\begin{tabular}{cccc}
\hline Sample & Mn/(g/mol $)$ & Mw/(g/mol $)$ & PDI \\
\hline $\mathrm{P}_{0}$ & 26,401 & 47,658 & 1.80 \\
$\mathrm{P}_{10}$ & 34,003 & 67,562 & 1.98 \\
$\mathrm{P}_{25}$ & 21,658 & 68,040 & 3.14 \\
$\mathrm{P}_{50}$ & 42,292 & 159,467 & 3.77 \\
$\mathrm{P}_{100}$ & 54,314 & 190,142 & 3.50 \\
\hline
\end{tabular}




\section{2. ${ }^{1} \mathrm{H}$ NMR characterization of copolymers}

Table S2. ${ }^{1} \mathrm{H}$ NMR data of P(MMA-co-BA)s

\begin{tabular}{|c|c|c|c|c|}
\hline \multirow{2}{*}{ Sample } & \multicolumn{2}{|c|}{ Theoretical Composition } & \multicolumn{2}{|c|}{ Calculation by ${ }^{1} \mathrm{H}$ NMR } \\
\hline & Percent $(\mathrm{mol} \%)^{a}$ & Proportion $^{b}$ & Percent $(\mathrm{mol} \%)^{a}$ & Proportion $^{b}$ \\
\hline $\mathrm{P}_{10}$ & $10 \%$ & 27 & $9.6 \%$ & 28.20 \\
\hline $\mathrm{P}_{25}$ & $25 \%$ & 9 & $28.1 \%$ & 7.65 \\
\hline $\mathrm{P}_{50}$ & $50 \%$ & 3 & $52.6 \%$ & 2.70 \\
\hline
\end{tabular}

${ }^{a}$ Mole percent of $\mathrm{BA}$ units in $\mathrm{P}(\mathrm{MMA}-\mathrm{co}-\mathrm{BA}) \mathrm{s} ;{ }^{b}$ Integral area ratio from theoretical composition and calculation by ${ }^{1} \mathrm{H}$ NMR. 


\subsection{In vitro degradation of copolymers}

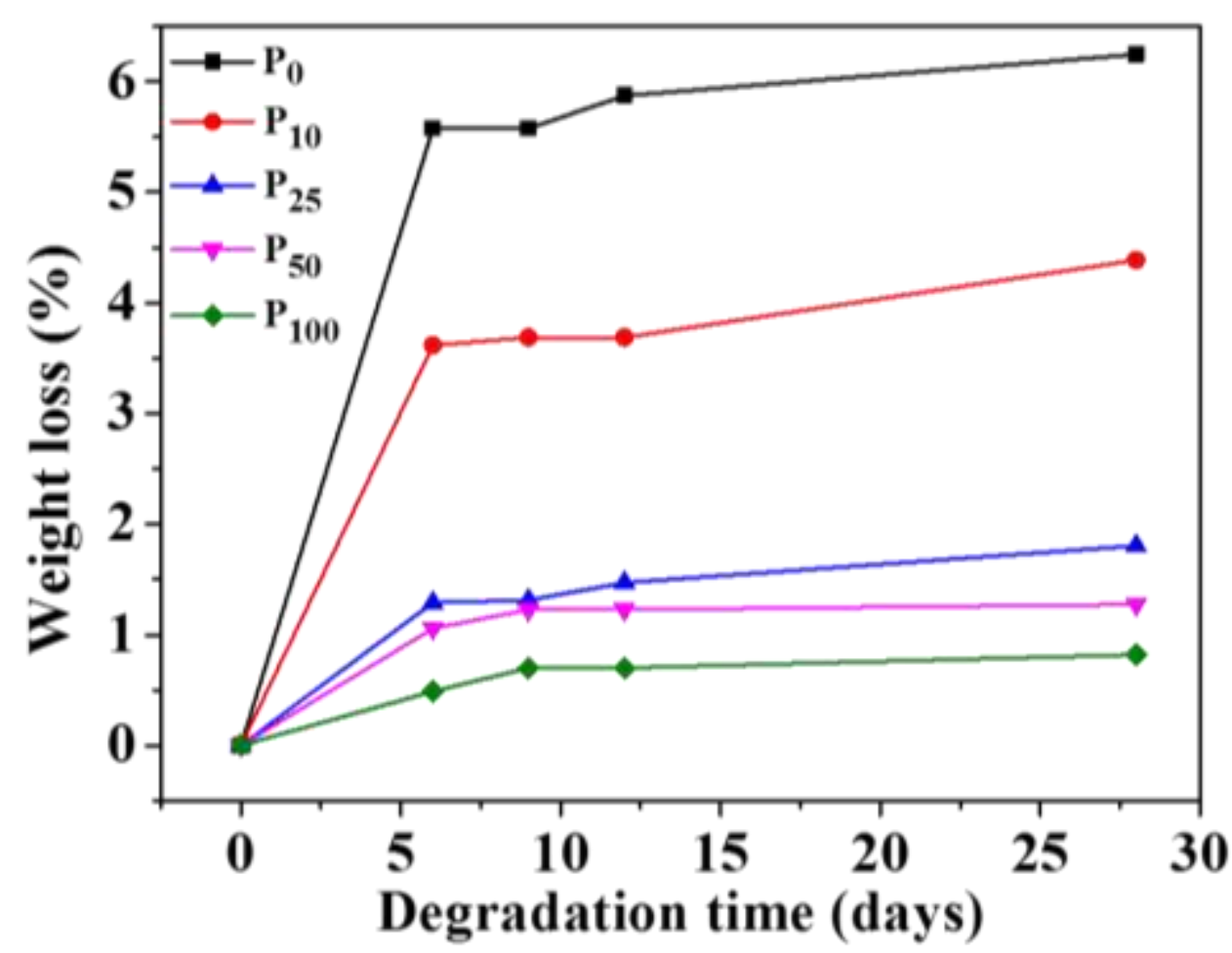

Figure S2. In vitro degradation of $\mathrm{P}\left(\mathrm{MMA}-\right.$ co-BA)s evaluated in $\mathrm{PBS}$ at $37^{\circ} \mathrm{C}$. For the first six days, those films displayed more weight loss due to the effect of sample edge in our opinion. After that, copolymers showed good stability since less than $1 \%$ weight loss was recorded for each test sample. 


\section{Antibacterial assessments}

\section{1. "Prison Break" experiments}

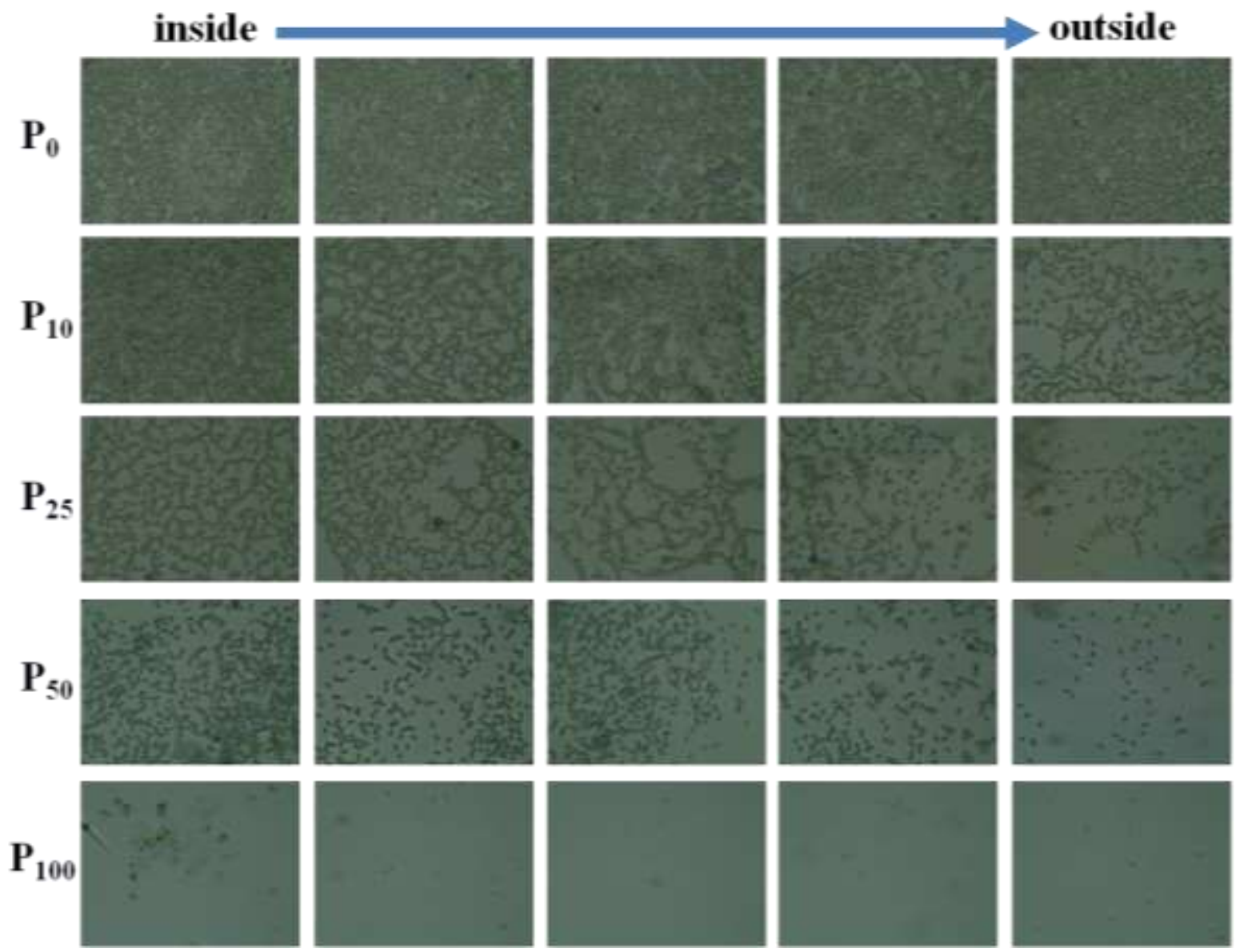

Figure S3. Optical micrographs of B. subtilis adhered on Px films from the inside (left) to outside (right) edges after $120 \mathrm{~h}$ of incubation. The image size is approximately $897 \mu \mathrm{m}^{2}(34.5 \mu \mathrm{m} \times 26.0 \mu \mathrm{m})$. 


\subsection{Plate count tests}


Figure S4. Plate count experiments of E. coli. Px films were soaked in an E. coli suspension for 24 h. $100 \mu \mathrm{L}$ dispersion was firstly diluted to $1 \mathrm{~mL}$, then $100 \mu \mathrm{L}$ of diluent was coated on beef-protein medium and further cultured for $24 \mathrm{~h}$ at $37^{\circ} \mathrm{C}$. Colony numbers corresponding to viable $E$. coli on $\mathrm{P}_{0}, \mathrm{P}_{10}, \mathrm{P}_{25}, \mathrm{P}_{50}$ and $\mathrm{P}_{100}$ films were 566, 245, 105, 6 and 0 units, respectively. 


\subsection{Optical density}

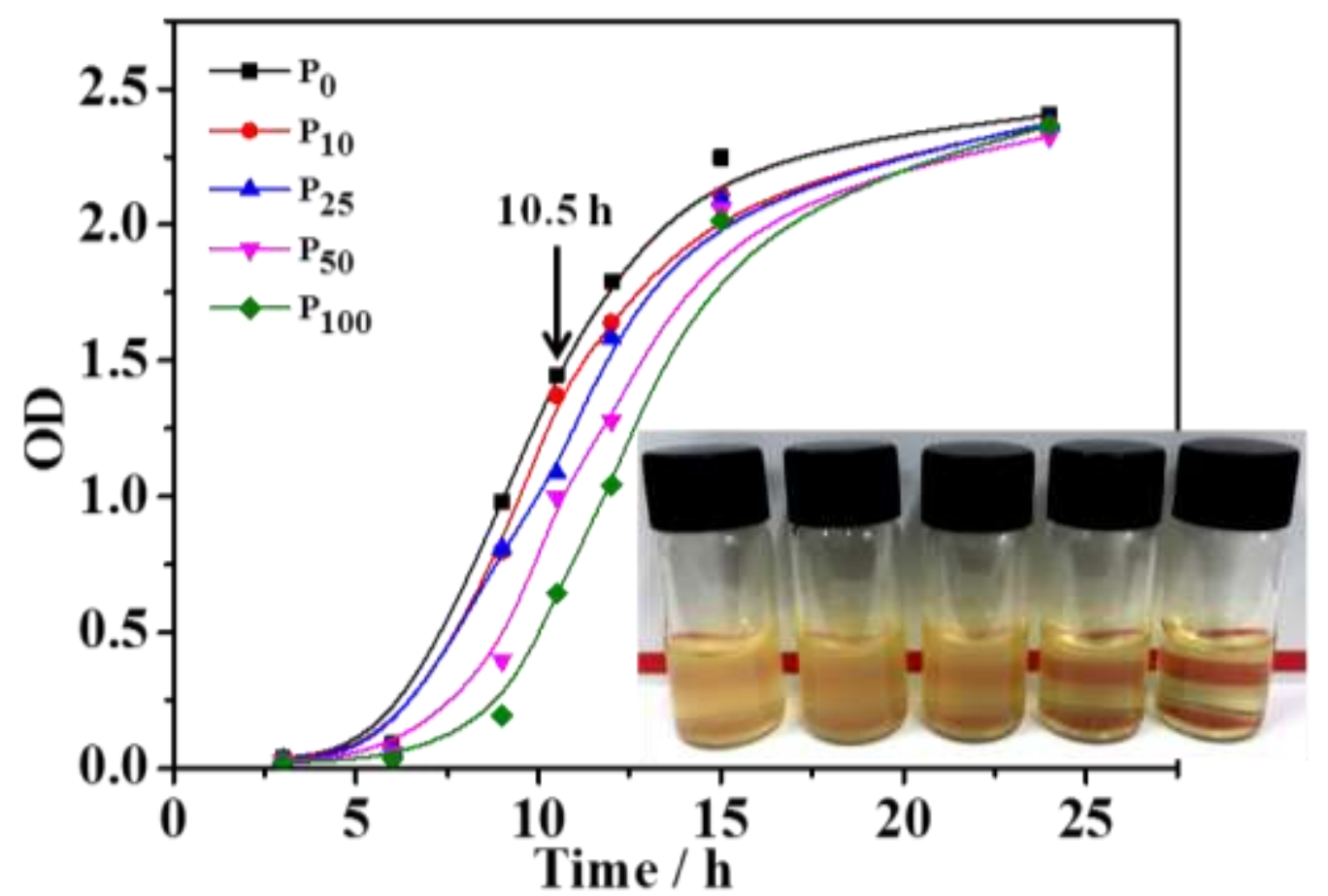

Figure S5. $\mathrm{OD}_{600}$ test for evaluating interactions between $E$. coli and $\mathrm{P}_{\mathrm{x}}$ films. Films were soaked in an E. coli suspension for $24 \mathrm{~h}$. After gently rinsing, they were transferred to fresh culture medium for the $\mathrm{OD}_{600}$ testing. The $E$. coli suspension displayed different turbidity at $10.5 \mathrm{~h}$ as shown in insert. 